\title{
Synthesis of New Amide and ThioUrease Compounds.
}

\author{
Waleed.F. Al-Hity *
}

Date of acceptance 5/2/2007

\begin{abstract}
:
The reaction of some new Schiff bases ( 2-[(2-Amino - ethylimino)-methyl]-R , 2-(\{2-[(R-benzylidene)-amino]-ethylimino $\}$-methyl $)-\mathrm{R}$ with Benzoyl chloride or Acetyl chloride were carried out. Subsequent reactions of these products

$\mathrm{N}$-(2-Amino-ethyl)-N-[Chloro-(R) -methyl]-benzamide or $\mathrm{N}-(2-\{\check{\mathrm{R}}-[$ chloro-(R) methyl]-amino $\}$-ethyl)-N-[chloro-(R) -methyl]- benzamide with thiourea afforded thioureas compounds.

The synthesized compounds were confirmed by their IR,UV,spectra and C.H.N. analysis.
\end{abstract}

\section{Introduction:}

It is known that Schiff bases react smoothly with acid chlorides and anhydrides to give the corresponding addition products ${ }^{[1-5]}$

Reaction of Schiff base with acid chloride gave imide compounds and reaction of imidecompounds with thiourea afforded thioureas compounds [6]

The synthesis of some new phenobarbital compounds from the reaction of some phenobarbital system containing Schiff bases moiety with benzoyl and 3,5-dinitro benzoyl chlorides .Subsequent reactionsof these products with thiourea afforded thioureas compounds ${ }^{[7]}$.

\section{Experimental:}

Melting points were recorded on Gallenkamp melting points Apparatus and were uncorrected . Elemental analysis was carried out in Mutah University on perkin-Elmre $2400 \mathrm{CHN}$ Elemental analyzer. FT-IR spectra were recorded on FT-IR spectrophotometer 8400s Shimadza (KBr) and UV-Visible spectra were recorded (in ethanol) On Schimadza $\quad$ Reco- 160 Spectrophotometer.

\section{Preparation of Schiff bases $(\mathbf{A}, \mathbf{B}, \mathbf{C}, \mathbf{D})$ :-}

Ethylimino and diethylimino were prepared by condensation of ethylene diamine with substituted benzaldehyde . To a solution of 0.05 mole of Ethylene diamine in $30 \mathrm{ml}$ of Ethanol (absolute) was added 0.05 mole or 0.1 mole of substituted benzaldehyde and refluxed $2 \mathrm{hr}$. Whereby ayellow crystalline solid separated out . The solid was filtered and recrystallized from ethanol. ${ }^{[8]}$.

Preparation of N-(2-Aminoethyl)-N-[Chloro-(R) -methyl]benzamide or acetamide :-

To an appropriate Schiff base (0.001 mole), Acetyl chloride or benzoyl chlorid ( 0.015 mole) in dry benzene ( 25 $\mathrm{ml})$ was added. The mixture was refluxed for ( $6 \mathrm{hrs}$ ), cooled, filtered and recrystallized from absolut ethanol ( Tables 1 ).

\section{Preparation of thioureas compounds:-}

To an appropriate derivatives of (1-13) (0.001mole) thiourea (0.002 mole) and $\mathrm{Na}_{2} \mathrm{CO}_{3}$ (0.002 mole) in absolute ethanol $(30 \mathrm{ml})$ were added . The mixture was refluxed for ( $3 \mathrm{hrs}$ ), cooled and filtered. The filtrate was

*Chem.Dept.College of Education for women, University of Al-Anbar 
poured into crushed ice, the separated solid was collected and recrystalized from 1,4-dioxan solvent ( Tables 2 ).

\section{Results and Discussion :-}

Schiff bases (A,B,C,D) were prepared by condensation of ethylene diamine with derivatives aldehyde ( $\mathrm{o}-$ hydroxy benzaldehyde , 4-dimethyl amino benzaldhyde). The reaction was followed by the appearance of absorption bands(1625-1645 $\mathrm{cm}^{-1}$ ) for $(v C=N)$ at in their IR spectra. In this work the reaction of Schiff-bases compounds with acetyl or benzoyl chlorides and subsequent reactions of above reaction products (1-13) with thiourea were carried out as shown in scheme (1).

However, treatment of Schiff bases with acid halides results in the formation of compounds (1-13) in which two groups $(\mathrm{Cl}, \mathrm{RC}=\mathrm{O})$ were introduced in the same step of the reaction. This reaction was followed by disappearance of absorption bands at

$\left(1235-1250 \mathrm{~cm}^{-1}\right)$ and $\left(720-760 \mathrm{~cm}^{-1}\right)$ which were attributed to $(\mathrm{C}-\mathrm{N})$ and $(\mathrm{C}$ Cl) moieties.

The reaction was involved the attack of the azomethine nitrogen by the carbonyl group of the aroyl chlorides, displacing the chloride as chloride anion and forming the iminium cation.

However, iminium cation was unstable, so the $\mathrm{Cl}-$ attacked $-+\mathrm{N}=\mathrm{C}$ moiety and afforded more stable covalently bonded compounds (1-13) Scheme 2).

Moreover, the reactions of acid halides addition products (1-13) with thiourea were afforded thioureas products (14-25). So, heating compounds (1-13) under reflux with thiourea in the presence of $\mathrm{Na}_{2} \mathrm{CO}_{3}$ for (3hrs) led to the nucleophilic substitution
(14 -25) were formed through the following mechanism (Scheme 3 ).

These compounds (14 -25) were characterized by their IR spectra. New doublet absorption bands in the region ( $\left.3250-3450 \mathrm{~cm}^{-1}\right)$ were attributed to $\left(\mathrm{NH}_{2}\right)$ and $(\mathrm{NH})$ functional moieties. Other characteristic bands in the region $\left(650-720 \mathrm{~cm}^{-1}\right)$ correlated to (v C-S) moiety. Moreover, (v C-Cl ) around ( $730-750 \mathrm{~cm}^{-1}$ ) disappeared.

They reveated strong absorption ranging from (224-235 )nm and from (240-290 )nm. these absorptions were due $\pi-\pi^{*}$ transition or $\eta$ - electrons of nitrogen atom which was in conjugation with neighbring groups.

The UV-Visibl spectra showed the following maxima (223-238)nm and (300-383)nm, due to the presence of aromatic rings and avariety of substituted grouos .

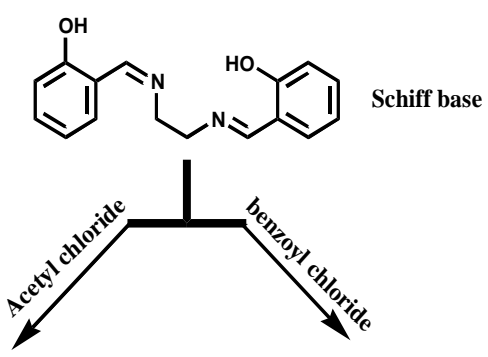

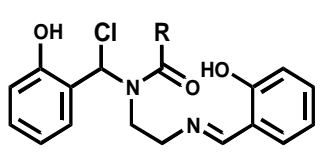

R = methyl
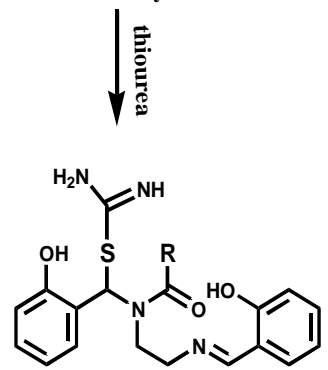

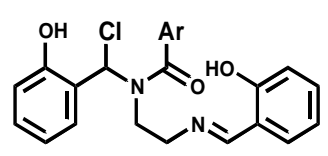

Ar $=$ phenyl

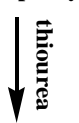<smiles>N=C(N)SC(c1ccccc1O)N(CC/N=C/c1ccccc1O)C(=O)O</smiles> 


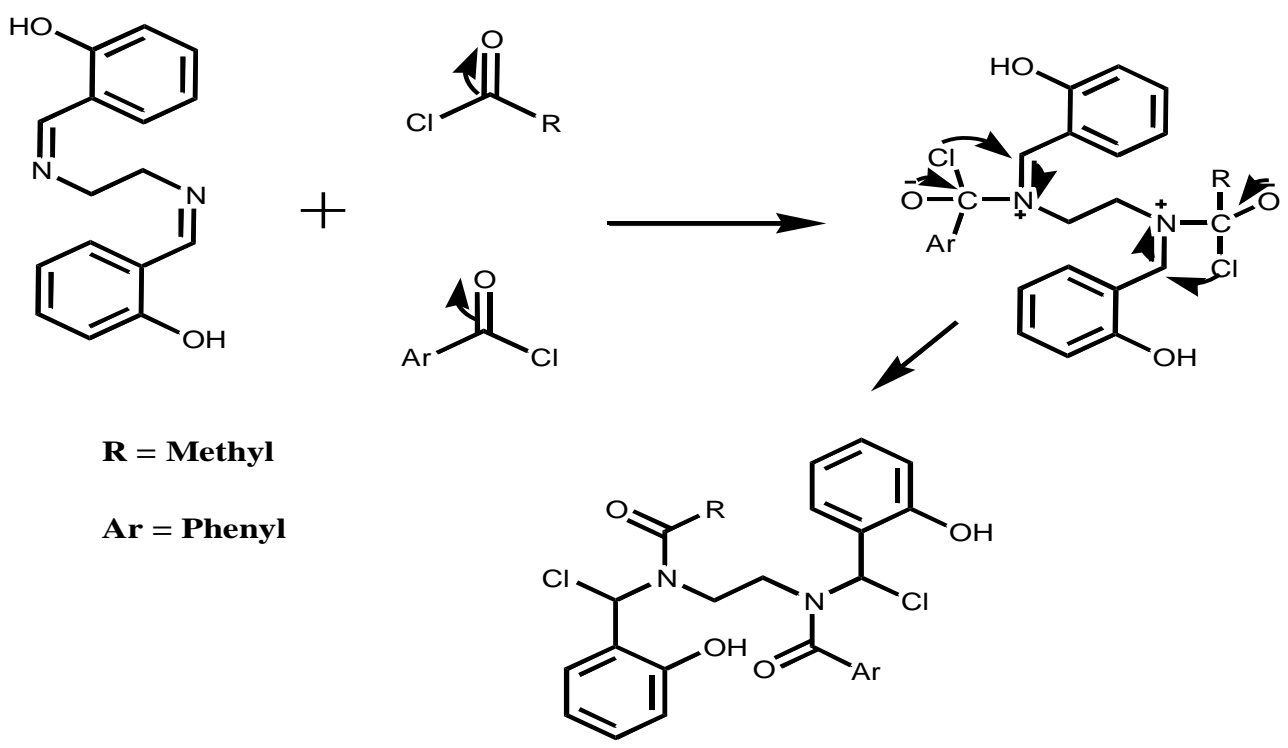

(Scheme 2)

(1)<smiles>NC(=C=C=C=S=C(N)N)CS</smiles>

(2)

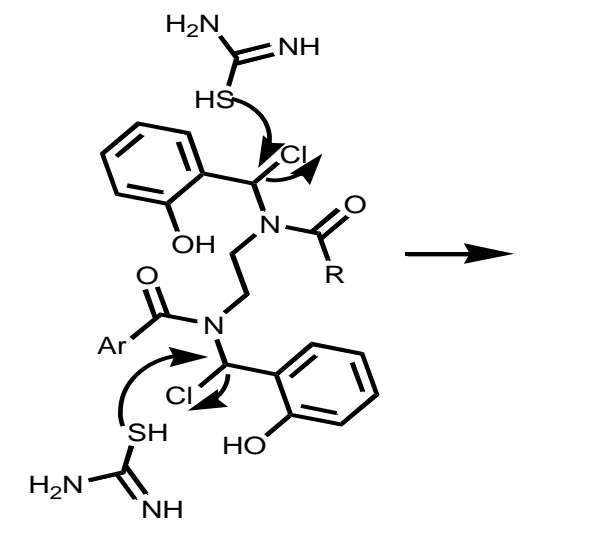<smiles>[R]C(=O)N(CCN(C(=O)[Ga])C(SC(=N)N)c1ccccc1O)C(SC(=N)N)c1ccccc1O</smiles>

(Scheme 3)

Table(1) : Some physical properties and C.H.N. analyses of compound (1-13).

\begin{tabular}{|c|c|c|c|c|c|c|c|c|c|c|}
\hline \multirow[b]{2}{*}{ Comp. } & \multirow[b]{2}{*}{$\mathbf{m} \cdot \mathbf{p} / \mathbf{C}^{0}$} & \multirow[b]{2}{*}{ Yield\% } & \multirow[b]{2}{*}{ Colour } & \multirow[b]{2}{*}{ M.F } & \multicolumn{3}{|c|}{ Calc. } & \multicolumn{3}{|c|}{ Found } \\
\hline & & & & & $\mathbf{C}$ & $\mathbf{H}$ & $\mathbf{N}$ & $\mathbf{C}$ & $\mathbf{H}$ & $\mathbf{N}$ \\
\hline 1 & 200 & 60 & orange & $\mathrm{C}_{16} \mathrm{H}_{17} \mathrm{ClN}_{2} \mathrm{O}_{2}$ & 63.05 & 5.62 & 9.19 & 62.95 & 5.56 & 9.00 \\
\hline 2 & 179 & 71 & yellow & $\mathrm{C}_{18} \mathrm{H}_{22} \mathrm{ClN}_{3} \mathrm{O}$ & 65.15 & 6.68 & 12.66 & 65.02 & 6.58 & 12.54 \\
\hline 3 & 221 & 55 & orange & $\mathrm{C}_{23} \mathrm{H}_{21} \mathrm{ClN}_{2} \mathrm{O}_{3}$ & 67.56 & 5.18 & 6.85 & 67.45 & 5.04 & 6.77 \\
\hline 4 & 228 & 58 & yellow & $\mathrm{C}_{25} \mathrm{H}_{26} \mathrm{ClN}_{3} \mathrm{O}_{2}$ & 68.88 & 6.01 & 9.64 & 68.75 & 6.00 & 9.61 \\
\hline 5 & 211 & 62 & orange & $\mathrm{C}_{30} \mathrm{H}_{26} \mathrm{Cl}_{2} \mathrm{~N}_{2} \mathrm{O}_{4}$ & 65.58 & 4.77 & 5.10 & 65.44 & 4.71 & 5.00 \\
\hline 6 & 230 & 68 & yellow & $\mathrm{C}_{32} \mathrm{H}_{31} \mathrm{Cl}_{2} \mathrm{~N}_{3} \mathrm{O}_{3}$ & 66.67 & 5.42 & 7.29 & 66.54 & 5.40 & 7.15 \\
\hline 7 & 171 & 52 & yellow & $\mathrm{C}_{34} \mathrm{H}_{36} \mathrm{C}_{12} \mathbf{N}_{4} \mathbf{O}_{2}$ & 67.66 & 6.01 & 9.28 & 67.58 & 5.98 & 9.20 \\
\hline 8 & 160 & 50 & white & $\mathrm{C}_{11} \mathrm{H}_{15} \mathrm{ClN}_{2} \mathrm{O}_{2}$ & 54.44 & 6.23 & 11.54 & 54.35 & 6.19 & 11.47 \\
\hline 9 & 157 & 73 & orange & $\mathrm{C}_{13} \mathrm{H}_{20} \mathrm{ClN}_{3} \mathrm{O}$ & 57.88 & 7.47 & 15.58 & 57.78 & 7.43 & 15.47 \\
\hline 10 & 181 & 66 & Brown & $\mathrm{C}_{20} \mathrm{H}_{22} \mathrm{Cl}_{2} \mathrm{~N}_{2} \mathrm{O}_{4}$ & 56.48 & 5.21 & 6.59 & 56.34 & 5.17 & 6.45 \\
\hline 11 & 192 & 60 & orange & $\mathrm{C}_{25} \mathrm{H}_{24} \mathrm{Cl}_{2} \mathrm{~N}_{2} \mathrm{O}_{4}$ & 61.61 & 4.96 & 5.75 & 61.55 & 4.87 & 5.68 \\
\hline 12 & 202 & 53 & yellow & $\mathrm{C}_{27} \mathrm{H}_{29} \mathrm{Cl}_{2} \mathrm{~N}_{3} \mathrm{O}_{3}$ & 63.04 & 5.68 & 8.17 & 62.98 & 5.52 & 8.07 \\
\hline 13 & 189 & 67 & white & $\mathrm{C}_{29} \mathrm{H}_{34} \mathrm{C}_{12} \mathrm{~N}_{4} \mathrm{O}_{2}$ & 64.32 & 6.33 & 10.35 & 64.21 & 6.23 & 10.19 \\
\hline
\end{tabular}


Table(2) : Some physical properties and C.H.N. analyses of compound (14-25).

\begin{tabular}{|c|c|c|c|c|c|c|c|c|c|c|}
\hline \multirow{2}{*}{ Comp. } & \multirow{2}{*}{ m.p/ $\mathbf{C}^{\mathbf{o}}$} & \multirow{2}{*}{ Yield\% } & \multirow{2}{*}{ Colour } & \multirow{2}{*}{ M.F } & \multicolumn{3}{|c|}{ Calc. } & \multicolumn{3}{|c|}{ Found } \\
\hline & & & & & C & $\mathbf{H}$ & $\mathbf{N}$ & $\mathbf{C}$ & $\mathbf{H}$ & $\mathbf{N}$ \\
\hline 14 & 149 & 51 & $\begin{array}{c}\text { Light } \\
\text { brown }\end{array}$ & $\mathrm{C}_{17} \mathrm{H}_{20} \mathrm{~N}_{4} \mathrm{O}_{2} \mathrm{~S}$ & 59.28 & 5.85 & 16.27 & 59.20 & 5.78 & 16.16 \\
\hline 15 & 177 & 55 & Brown & $\mathrm{C}_{19} \mathrm{H}_{25} \mathrm{~N}_{5} \mathrm{OS}$ & 61.43 & 6.78 & 18.85 & 61.33 & 6.67 & 18.75 \\
\hline 16 & 185 & 46 & Orange & $\mathrm{C}_{24} \mathrm{H}_{24} \mathrm{~N}_{4} \mathrm{O}_{3} \mathrm{~S}$ & 64.27 & 5.39 & 12.49 & 64.11 & 5.26 & 12.40 \\
\hline 17 & 196 & 49 & Brown & $\mathrm{C}_{26} \mathrm{H}_{29} \mathrm{~N}_{5} \mathrm{O}_{2} \mathrm{~S}$ & 65.66 & 6.15 & 14.73 & 65.53 & 6.12 & 14.66 \\
\hline 18 & 242 & 62 & yellow & $\mathrm{C}_{32} \mathrm{H}_{32} \mathrm{~N}_{6} \mathrm{O}_{4} \mathrm{~S}_{2}$ & 61.13 & 5.13 & 13.37 & 61.05 & 5.07 & 13.24 \\
\hline 19 & 233 & 70 & yellow & $\mathrm{C}_{36} \mathrm{H}_{42} \mathrm{~N}_{8} \mathrm{O}_{2} \mathrm{~S}_{2}$ & 63.32 & 6.20 & 16.41 & 63.25 & 6.12 & 16.35 \\
\hline 20 & 162 & 54 & Orange & $\mathrm{C}_{12} \mathrm{H}_{18} \mathrm{~N}_{4} \mathrm{O}_{2} \mathrm{~S}$ & 51.04 & 6.43 & 19.84 & 50.89 & 6.32 & 19.72 \\
\hline 21 & 140 & 50 & Brown & $\mathrm{C}_{14} \mathrm{H}_{23} \mathrm{~N}_{5} \mathrm{OS}$ & 54.34 & 7.49 & 22.63 & 54.27 & 7.38 & 22.50 \\
\hline 22 & 226 & 45 & Orange & $\mathrm{C}_{22} \mathrm{H}_{28} \mathrm{~N}_{6} \mathrm{O}_{4} \mathrm{~S}_{2}$ & 52.36 & 5.59 & 16.65 & 52.28 & 5.48 & 16.51 \\
\hline 23 & 211 & 41 & yellow & $\mathrm{C}_{29} \mathrm{H}_{35} \mathrm{~N}_{7} \mathrm{O}_{3} \mathrm{~S}_{2}$ & 58.66 & 5.94 & 16.51 & 58.52 & 6.00 & 16.49 \\
\hline 24 & 228 & 52 & Brown & $\mathrm{C}_{31} \mathrm{H}_{40} \mathrm{~N}_{8} \mathrm{O}_{2} \mathrm{~S}_{2}$ & 59.97 & 6.49 & 18.05 & 59.88 & 6.40 & 17.89 \\
\hline 25 & 205 & 50 & yellow & $\mathrm{C}_{31} \mathrm{H}_{40} \mathrm{~N}_{8} \mathrm{O}_{2} \mathrm{~S}_{2}$ & 57.22 & 5.34 & 14.83 & 57.12 & 5.23 & 14.70 \\
\hline
\end{tabular}

Table(3): IR Spectral data of Compounds (1-13).

\begin{tabular}{|c|c|c|c|c|c|c|c|c|c|}
\hline \multicolumn{10}{|c|}{ Characteristic bands of IR spactrum } \\
\hline $\begin{array}{c}\text { Com. } \\
\text { No. }\end{array}$ & $\begin{array}{c}\text { v }(\mathrm{O}-\mathrm{H}) \\
\left(\mathrm{cm}^{-1}\right)\end{array}$ & $\begin{array}{c}\boldsymbol{v}(\mathbf{C}-\mathbf{H}) \\
\operatorname{arom} \\
\left(\mathbf{c m}^{-1}\right)\end{array}$ & $\begin{array}{c}\text { v }(\mathbf{C}-\mathbf{H}) \\
\text { aliph. } \\
\left(\mathrm{cm}^{-1}\right)\end{array}$ & $\begin{array}{c}v(C=O) \\
\left(\mathrm{cm}^{-1}\right)\end{array}$ & $\begin{array}{c}v(C=C) \\
\left(\mathrm{cm}^{-1}\right)\end{array}$ & $\begin{array}{c}v(\mathrm{C}-\mathrm{O}) \\
\left(\mathrm{cm}^{-1}\right)\end{array}$ & $\begin{array}{c}v(\mathrm{C}-\mathrm{N}) \\
\left(\mathrm{cm}^{-1}\right)\end{array}$ & $\begin{array}{c}\text { v }(\mathrm{C}-\mathrm{Cl} \\
\left(\mathrm{cm}^{-1}\right)\end{array}$ & $\begin{array}{c}v(C=N) \\
\left(\mathrm{cm}^{-1}\right)\end{array}$ \\
\hline 1 & 3430 & 3065 & 2870 & 1680 & 1580 & 1255 & 1220 & 750 & - \\
\hline 2 & - & 3070 & 2850 & 1690 & 1570 & 1270 & 1225 & 730 & - \\
\hline 3 & 3450 & 3090 & 2880 & 1685 & 1570 & 1290 & 1230 & 730 & 1625 \\
\hline 4 & - & 3080 & 2890 & 1680 & 1595 & 1260 & 1240 & 740 & 1630 \\
\hline 5 & 3445 & 3080 & 2890 & 1690 & 1580 & 1280 & 1230 & 740 & - \\
\hline 6 & 3450 & 3090 & 2870 & 1675 & 1560 & 1250 & 1210 & 740 & - \\
\hline 7 & - & 3070 & 2875 & 1690 & 1555 & 1265 & 1230 & 730 & - \\
\hline 8 & 3440 & 3080 & 2900 & 1680 & 1570 & 1270 & 1240 & 745 & - \\
\hline 9 & - & 3060 & 2930 & 1680 & 1580 & 1270 & 1235 & 750 & - \\
\hline 10 & 3450 & 3050 & 2870 & 1690 & 1585 & 1280 & 1240 & 740 & - \\
\hline 11 & 3430 & 3070 & 2880 & 1685 & 1570 & 1290 & 1240 & 730 & - \\
\hline 12 & 3440 & 3090 & 2890 & 1675 & 1595 & 1275 & 1220 & 740 & - \\
\hline 13 & - & 3060 & 2930 & 1680 & 1590 & 1270 & 1230 & 750 & - \\
\hline
\end{tabular}

Table(4): IR Spectral data of Compounds (14-25).

\begin{tabular}{|c|c|c|c|c|c|c|c|c|c|}
\hline \multicolumn{10}{|c|}{ Characteristic bands of IR spactrum } \\
\hline $\begin{array}{c}\text { Comp. } \\
\text { No. }\end{array}$ & $\begin{array}{c}\text { v }(\mathrm{O}-\mathrm{H}) \\
\left(\mathrm{cm}^{-1}\right)\end{array}$ & $\begin{array}{c}v(\mathrm{NH}) \\
\left(\mathrm{cm}^{-1}\right)\end{array}$ & $\begin{array}{c}\text { v }(\mathbf{C}-\mathbf{H}) \\
\text { arom } \\
\left(\mathbf{c m}^{-1}\right)\end{array}$ & $\begin{array}{c}\text { v }(\mathbf{C}-\mathbf{H}) \\
\text { aliph. } \\
\left(\mathbf{c m}^{-1}\right)\end{array}$ & $\begin{array}{c}v(C=O) \\
\left(\mathrm{cm}^{-1}\right)\end{array}$ & $\begin{array}{c}v(C=N) \\
\left(\mathrm{cm}^{-1}\right)\end{array}$ & $\begin{array}{c}v(C=C) \\
\left(\mathrm{cm}^{-1}\right)\end{array}$ & $\begin{array}{c}v(C-S) \\
\left(\mathrm{cm}^{-1}\right)\end{array}$ & $\begin{array}{c}v(\mathrm{C}-\mathrm{N}) \\
\left(\mathrm{cm}^{-1}\right)\end{array}$ \\
\hline 14 & 3440 & 3200 & 3060 & 2870 & 1680 & - & 1540 & 1260 & 1220 \\
\hline 15 & - & 3230 & 3080 & 2890 & 1670 & - & 1560 & 1265 & 1230 \\
\hline 16 & 3445 & 3300 & 3070 & 2880 & 1665 & 1635 & 1570 & 1250 & 1235 \\
\hline 17 & 3440 & 3260 & 3050 & 2890 & 1670 & 1630 & 1590 & 1280 & 1230 \\
\hline 18 & 3450 & 3250 & 3060 & 2870 & 1660 & - & 1545 & 1270 & 1240 \\
\hline 19 & - & 3300 & 3050 & 2860 & 1680 & - & 1540 & 1260 & 1245 \\
\hline 20 & 3450 & 3240 & 3070 & 2895 & 1685 & - & 1550 & 1260 & 1240 \\
\hline 21 & - & 3230 & 3090 & 2880 & 1670 & - & 1580 & 1250 & 1230 \\
\hline 22 & 3445 & 3200 & 3090 & 2870 & 1680 & - & 1560 & 1260 & 1220 \\
\hline 23 & 3450 & 3240 & 3060 & 2880 & 1685 & - & 1585 & 1250 & 1225 \\
\hline 24 & - & 3240 & 3050 & 2870 & 1670 & - & 1575 & 1290 & 1230 \\
\hline 25 & 3450 & 3200 & 3080 & 2860 & 1670 & - & 1560 & 1280 & 1240 \\
\hline
\end{tabular}


Table(5): UV Spectral data of Compounds (1-25).

\begin{tabular}{|c|c|}
\hline Comp.No & UV-Visible absorption maxima $\lambda / \mathrm{nm}$ \\
\hline 1 & $311,278,269,233,226$ \\
\hline 2 & $370,320,266,252,225$ \\
\hline 3 & $365,300,260,241,220$ \\
\hline 4 & $360,300,261,298,226$ \\
\hline 6 & $377,300,269,235,220$ \\
\hline 7 & $380,316,272,293,222$ \\
\hline 8 & $382,319,265,259,230$ \\
\hline 9 & $372,301,265,244,225$ \\
\hline 10 & $375,301,275,236,221$ \\
\hline 11 & $340,300,255,230,220$ \\
\hline 12 & $345,302,266,239,223$ \\
\hline 13 & $349,299,256,241,228$ \\
\hline 14 & $341,288,251,244,220$ \\
\hline 15 & $361,305,249,236,221$ \\
\hline 16 & $358,295,246,236,222$ \\
\hline 17 & $346,300,250,229,221$ \\
\hline 18 & $339,306,252,229,223$ \\
\hline 19 & $350,306,260,241,230$ \\
\hline 20 & $356,505,285,240,228$ \\
\hline 21 & $352,300,271,236,222$ \\
\hline 22 & $358,310,277,236,224$ \\
\hline 23 & $354,311,274,233,228$ \\
\hline 24 & $383,320,266,230,226$ \\
\hline 25 & $377,310,255,231,220$ \\
\hline & $354,300,251,231,228$ \\
\hline
\end{tabular}

\begin{tabular}{|c|c|c|}
\hline No. & Name of compounds & Structure \\
\hline $\mathbf{A}$ & $\begin{array}{l}\text { 2-[(2-Amino-ethylimino)- } \\
\text { methyl]-phenol }\end{array}$ & $\mathrm{HO}_{2}$ \\
\hline $\mathbf{B}$ & 4-[(2-amino-ethyliminomethyl]aniline & \\
\hline $\mathbf{C}$ & $\begin{array}{l}\text { 2-(\{2-[(2-hydroxy-benzylidene)-amino] } \\
\text {-ethylimino\}-methyl)-phenol }\end{array}$ & \\
\hline $\mathbf{D}$ & $\begin{array}{l}\text { N-(4-Dimethylamino-benzylidene)-N'-(4- } \\
\text { Dimethylamino-benzylidene)-ethane-1,2 } \\
\text {-diamine }\end{array}$ & \\
\hline 1 & $\begin{array}{l}\text { N-(2-Amino-ethyl)-N-[chloro-(2-hydroxy- } \\
\text { phenyl)-methyl]-benzamide }\end{array}$ & \\
\hline 2 & $\begin{array}{l}\text { N-(2-Amino-ethyl)-N-[chloro-(4-dimethylamino- } \\
\text { phenyl)-methyl]-benzamide }\end{array}$ & \\
\hline $\mathbf{3}$ & $\begin{array}{c}\text { N-[Chloro-(2-hydroxy-phenyl)-methyl]-N- } \\
\{2-[(2-h y d r o x y-b e n z y l i d e n e)-a m i n o]-e t h y l\} \\
\text { benzamide }\end{array}$ & \\
\hline 4 & $\begin{array}{l}\text { N-[Chloro-(2-hydroxy-phenyl)-methyl]-N-\{2- } \\
\text { [(4-dimethylamino-benzylidene)-amino]- ethyl\}- } \\
\text { benzamide }\end{array}$ & \\
\hline 5 & $\begin{array}{l}\text { N-(2-\{Benzoyl-[chloro-(2-hydroxy-phenyl)-methyl]-amino\}- } \\
\text { ethyl)-N-[chloro-(2-hydroxy-phenyl)-methyl]-benzamide }\end{array}$ & \\
\hline
\end{tabular}




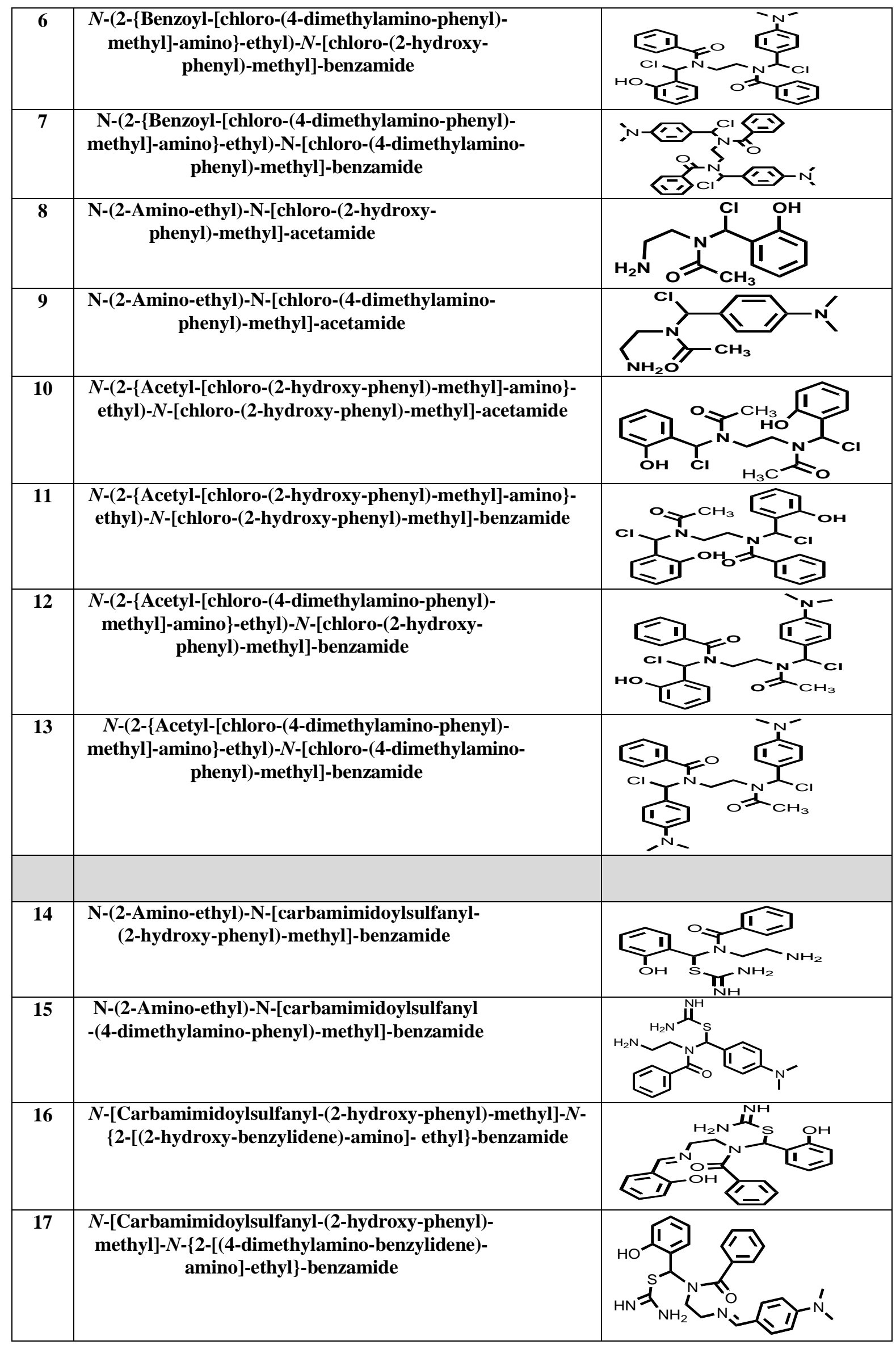




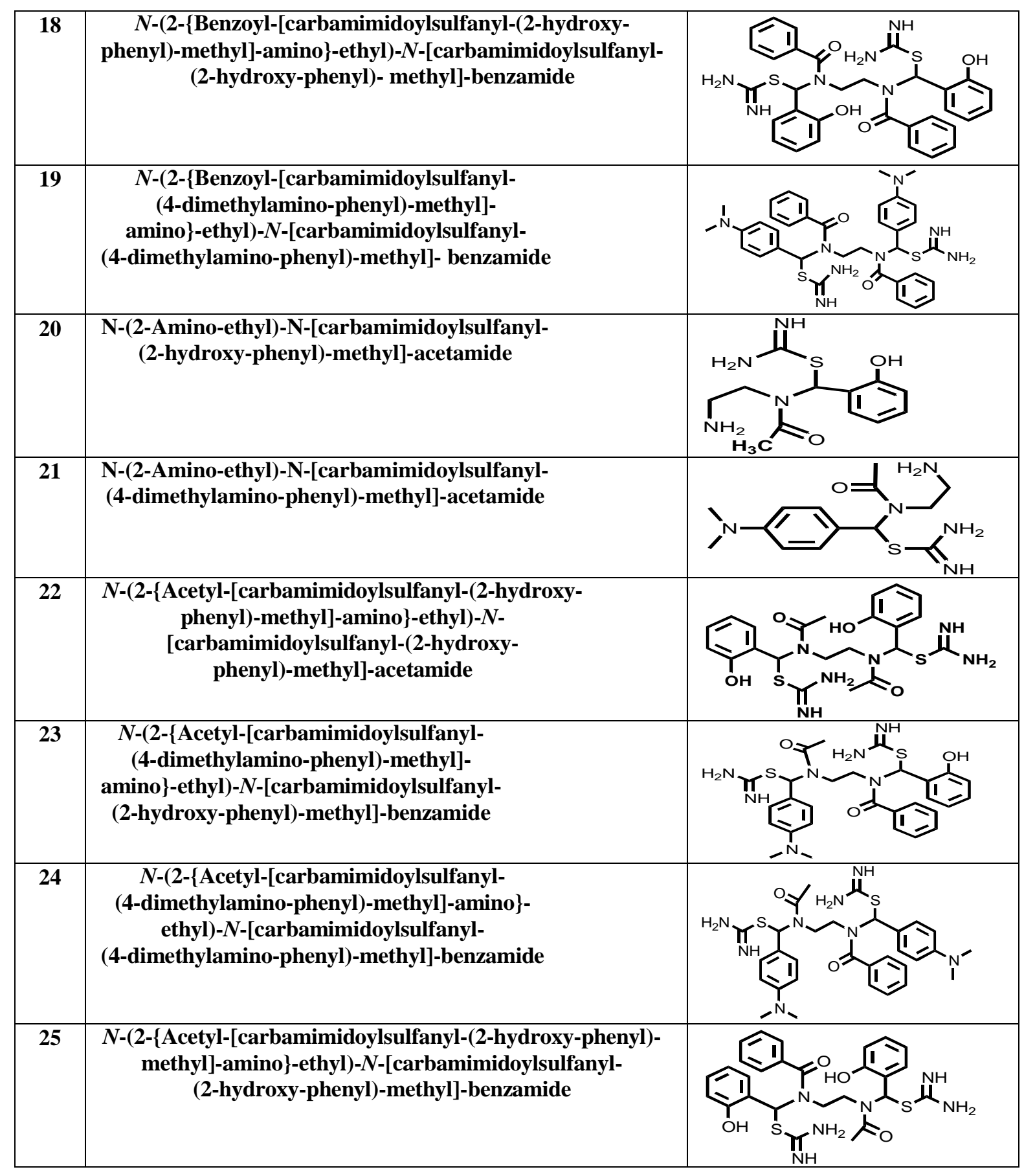

\section{REFERENCES:}

1-Takashi,T., Takeo, T., and Yashizo, S., 1978. Synthesis and cycloaddition reaction of di and tri- substituted 1,3oxazepine. Heterocycles, (11) : 331-6. 2-Tyoji, T., Kuniyoshi, I., and Takashi, T., 1987 . Photochemical and thermal reactions of some heterocyclic containing $\mathrm{C}=\mathrm{N}-\mathrm{O}$ and $\mathrm{N}=\mathrm{C}-\mathrm{o}$ group . Chem. Pharm. Bull., $35(8): 74$.

3- Biginelli,P.,Gazz., 1983 . New protocol for Biginelli reaction -a practical synthesis of Monastrol . Chim. Ital., 23 (3): 360.

4- Lin,H.X.,;Zhang,X.;Cheng,L.S., 1999 . Spectral analysis of organic compounds Chin.Chem.Lett.,10 (11) : 915-916. 
5- Hussein ,F.A., 2000. .Synthesis of Nsubstituted saccharin's via Schiff Bases. Iraqi

Journal of Chemistry $26(1): 42-50$.. 6- Hussein,F.A., Ali I.T. and Hassa ,D.F., 2001. Synthesis and characterization of 2-Aryl-3- phenyl-2,3Dihydro-1,3-oxazepine-4,7-Diones. Iraqi J. of Chem., 27 (2):445.

7- AL-Bayati ,R.I., Muslih ,R.M. and Janabiy, N., 2005 . Synthesis of new 5Ethyl 5- phenyl Barbituric Acid Derivatives . National Journal of Chemistry, 17(1) :138-142.

8-AL-Hity ,W.F.,AL- AL-Hadithi M. A, 2005 ( Synthesis and caracterization of oxazepine oxazepane from reaction of ( Schiff bases with maleic and succinic anhydride . Journal Of Al-Nahrain university vol . $8(2): 27-34$.

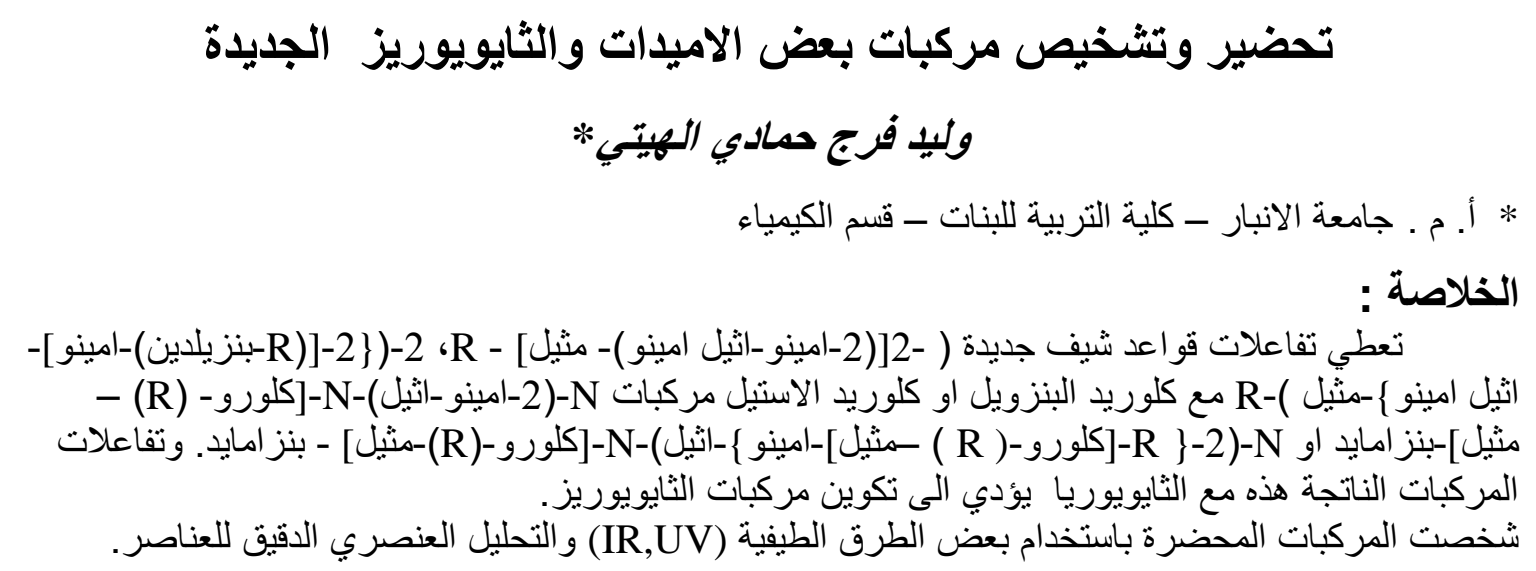

